\title{
Mortalidade associada ao uso da digoxina no tratamento da fibrilação atrial: uma revisão integrativa
}

\author{
Mortality associated with the use of digoxin in the treatment of atrial fibrillation: an integrative \\ review \\ Mortalidad asociada al uso de digoxina en el tratamiento de la fibrilación auricular: una revisión \\ integradora
}

\section{Resumo}

A fibrilação atrial (FA) é a arritmia mais prevalente, desencadeadora de um ritmo cardíaco irregular e acelerado. Embora nem sempre seja o fármaco de primeira escolha, a digoxina ainda é bastante usada, apesar de haver uma linha tênue entre seus efeitos terapêuticos, adversos e a mortalidade. Diante disso, o objetivo desse estudo é buscar evidências clínicas sobre a correlação entre o uso da digoxina e a mortalidade em pacientes com FA. Assim, foi realizada uma pesquisa bibliográfica, utilizando os descritores "(Digoxin) AND (Atrial fibrillation) AND (drug therapy)", nas bases de dados PubMed, Biblioteca Virtual em Saúde (BVS), Scientific Eletronic Library Online (Scielo) e Cochrane. A busca foi realizada durante o mês de fevereiro de 2021. Após a aplicação dos critérios de inclusão definidos, foram selecionados 15 estudos. Após análise, evidenciou-se o número crescente de estudos que relacionam a mortalidade à terapêutica com digoxina em paciente com FA e em associação a comorbidades, como insuficiência cardíaca (IC). Logo, embora não haja um consenso, a mortalidade foi superior nos grupos de portadores de FA, com cardioversor desfibrilador ou não, que utilizaram a digoxina; sendo a mortalidade ainda maior em idosos, em portadores de doenças renais, em pacientes com FA sem IC e na ausência de monitorização terapêutica. Diante das evidências apresentadas nessa revisão, podemos afirmar que o uso de digoxina em pacientes com FA deve ser evitado ou feito com cautela e acompanhamento rigoroso.

Palavras-chave: Digoxina; Fibrilação atrial; Tratamento farmacológico.

\section{Abstract}

Atrial fibrillation (AF) is the most prevalent arrhythmia, triggering an irregular and fast heart rhythm. Although it is not always the drug of first choice, digoxin is still widely used, although there is a fine line between its therapeutic effects, adverse effects, and mortality. Therefore, the aim of this study is to seek clinical evidence on the correlation 
between the use of digoxin and mortality in patients with AF. Thus, a literature search was conducted using the descriptors "(Digoxin) AND (Atrial fibrillation) AND (drug therapy)" in the PubMed, Virtual Health Library (VHL), Scientific Electronic Library Online (Scielo) and Cochrane databases. The search was conducted during the month of February 2021. After applying the defined inclusion criteria, 15 studies were selected. After analysis, it was evident the increasing number of studies that relate mortality to digoxin therapy in patients with AF and in association with comorbidities, such as heart failure (HF). Therefore, although there is no consensus, mortality was higher in groups of patients with AF, with or without cardioverter defibrillator, who used digoxin; mortality was even higher in the elderly, in patients with kidney disease, in patients with AF without HF and in the absence of therapeutic monitoring. Given the evidence presented in this review, we can state that the use of digoxin in patients with AF should be avoided or undertaken with caution and close monitoring.

Keywords: Digoxin, Atrial fibrillation, Drug therapy.

\section{Resumen}

La fibrilación auricular (FA) es la arritmia más frecuente, que provoca un ritmo cardíaco irregular y acelerado. Aunque no siempre es el fármaco de primera elección, la digoxina se sigue utilizando ampliamente, aunque existe una fina línea entre sus efectos terapéuticos y adversos y la mortalidad. Por ello, el objetivo de este estudio es buscar evidencia clínica sobre la correlación entre el uso de digoxina y la mortalidad en pacientes con FA. Así, se realizó una búsqueda bibliográfica utilizando los descriptores "(Digoxin) AND (Atrial fibrillation) AND (drug therapy)" en las bases de datos PubMed, Biblioteca Virtual em Saúde (BVS), Scientific Eletronic Library Online (Scielo) y Cochrane. La búsqueda se realizó durante el mes de febrero de 2021. Tras aplicar los criterios de inclusión definidos, se seleccionaron 15 estudios. Tras el análisis, fue evidente el creciente número de estudios que relacionan la mortalidad con el tratamiento con digoxina en pacientes con FA y en asociación con comorbilidades, como la insuficiencia cardíaca (IC). Por lo tanto, aunque no hay consenso, la mortalidad fue mayor en los grupos de pacientes con FA, con desfibrilador cardioversor o no, que utilizaron digoxina; la mortalidad fue incluso mayor en los ancianos, los pacientes con enfermedad renal, los pacientes con FA sin IC y en ausencia de monitorización terapéutica. Dadas las pruebas presentadas en esta revisión, podemos afirmar que el uso de digoxina en pacientes con FA debe evitarse o realizarse con precaución y estrecha vigilancia.

Palabras clave: Digoxina; Fibrilación auricular; Quimioterapia.

\section{Introdução}

A fibrilação atrial (FA) é a arritmia cardíaca mais comum na prática clínica, sendo caracterizada por um ritmo cardíaco irregular e acelerado (Chugh et al., 2014). No ano de 2010, uma estimativa global apontou a existência de 33.5 milhões de pessoas com fibrilação atrial e dados mais recentes, para o ano de 2016, apontam a presença de cerca de 46.3 milhões de indivíduos com a condição, de forma que tal crescimento na incidência e prevalência vem sendo observado e reportado também por outros estudos, atribuindo-se isso a fatores como o crescimento na expectativa de vida e a maior presença de comorbidades crônicas (Benjamin et al., 2019; Chugh et al., 2014; de Moraes et al., 2019; Go et al., 2001; Kornej, Börschel, Benjamin \& Schnabel, 2020).

Nesse sentido, verifica-se que a FA é um problema de saúde pública importante, relacionado a aumento de morbimortalidade, bem como a maior ônus econômico, de forma que é necessário atentar para as medidas preventivas da doença, mas também para a relação entre benefício, custo e risco das atuais opções terapêuticas, inserindo-se nesse campo a digoxina (Go et al., 2001; Rahman, Kwan \& Benjamin, 2014; Zoni-Berisso, Lercari, Carazza \& Domenicucci, 2014). Tal medicação corresponde a um glicosídeo cardíaco, cujo mecanismo de ação envolve a inibição da bomba Na+/K+ ATPase, gerando aumento da força de contração no coração e diminuição da frequência cardíaca, retardando a condução elétrica no nó atrioventricular (Ren et al, 2020).

No caso da FA, a escolha da digoxina como opção terapêutica vem sendo colocada em debate em razão de estudos que associam o uso do medicamento à maior mortalidade e outros desfechos desfavoráveis. Nesse âmbito, observa-se uma diminuição na utilização da digoxina, que, todavia ainda é bastante comum para o tratamento da FA, em boa parte dos casos, devido a seu longo histórico para controle da doença, bem como em razão das alternativas farmacológicas não possuírem segurança consolidada em relação à mortalidade (Somberg, 2014; Goldberger \& Alexander, 2014; Gao et al., 2019). Vale destacar que a European Society of Cardiology, em guideline de 2020, permanece indicando a utilização da digoxina para 
melhoria do prognóstico dos pacientes e destaca que os estudos contrários podem ser resultantes de vieses de seleção e prescrição (Hindricks et al., 2020). Os guidelines norte-americanos de 2014 e 2019 também indicam o uso do medicamento em algumas circunstâncias, ponderando sobre o risco de maior mortalidade apontado por certas pesquisas (January et al., 2014; January et al. 2019).

Dado o exposto, observa-se que a digoxina continua sendo uma das principais escolhas medicamentosas para o tratamento da fibrilação atrial, doença cardíaca com projeção de crescimento. Dessa forma, reconhecendo-se os impactos da FA para o desenvolvimento e/ou agravamento de outras comorbidades, redução da qualidade de vida e aumento com os gastos em saúde, o presente trabalho objetivou realizar um levantamento bibliográfico a fim de analisar estudos acerca da associação do uso da digoxina com a mortalidade em pacientes com fibrilação atrial.

\section{Metodologia}

O presente estudo trata-se de revisão integrativa que consiste em um método de pesquisa pautado na síntese de vários estudos, selecionados a partir de critérios bem definidos e pré-estabelecidos, possibilitando uma conclusão ampla acerca de uma determinada temática. Sendo assim, esta foi realizada de acordo com seis etapas, as quais consistem em: estabelecimento do tema e objetivo, seleção dos estudos mediante estabelecimento de critérios de inclusão e exclusão, formação do banco de dados, análise dos estudos escolhidos, interpretação dos resultados e apresentação da síntese do conhecimento (Souza, Silva \& Carvalho, 2010).

A questão norteadora formulada para revisão integrativa foi a seguinte: Qual a associação da digoxina com a mortalidade no tratamento medicamentoso de pacientes com fibrilação atrial? Após isso, a busca de estudos primários se deu a partir da utilização de descritores controlados do Medical Subject Headings (MeSH) e Descritores em Ciências de Saúde (DeCS), com os termos Digoxin, Atrial fibrillation e Drug therapy. Foi realizada a busca nas bases de dados hospedadas na Biblioteca Virtual em Saúde (BVS), além da Scientific Eletronic Library Online (Scielo), PubMed e Cochrane, durante o mês de fevereiro 2021 Os descritores foram combinados utilizando o operador booleano AND, na forma "Digoxin" AND "Atrial fibrillation" AND "Drug therapy".

Os filtros usados nas bases de dados englobaram publicações escritas na língua inglesa, portuguesa ou espanhola, publicadas nos últimos 5 anos. Foram elencados como critérios de inclusão do estudo, artigos relacionados ao tema e com texto completo disponível de modo gratuito. Por outro lado, os critérios de exclusão da amostra foram: editoriais, cartas ao editor, relatos de caso, guias e revisões, bem como estudos que não incluíssem o objeto de pesquisa.

\section{Resultados}

As pesquisas resultaram em uma amostra inicial de 119 artigos. Em seguida, pela análise dos títulos foram selecionados 65 artigos. Após isso, as leituras de seus resumos repercutiram na limitação de 29 artigos para serem lidos na íntegra. Por fim, a análise completa desses últimos estudos resultou em uma coletânea final de 15 artigos, com discussões inseridas dentro da temática proposta, os quais foram selecionados para compor esta revisão integrativa. Segue o fluxograma de estratégia de busca (Figura 1). 
Figura 1. Fluxograma representando a seleção de artigos de acordo com as recomendações PRISMA.

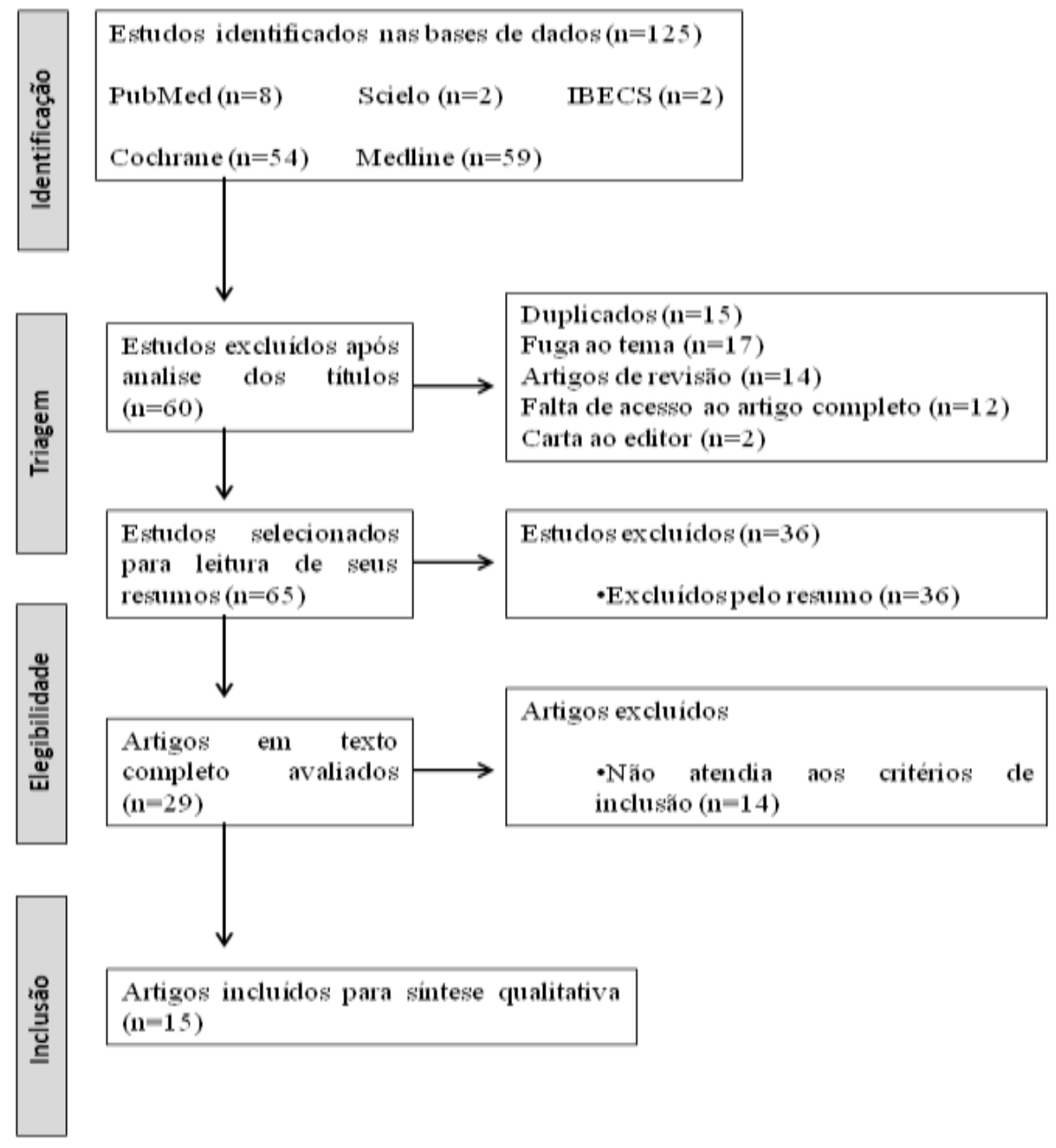

Fonte: Autores (2020)

Decorrente da leitura integral dos 15 artigos selecionados foi feita a categorização, análise e interpretação dos achados, bem como a apresentação e divulgação dos resultados. Foram contemplados os seguintes dados: periódico, ano de publicação, autor, base de dados, idioma e principal achado, como registrado nos Quadros 1 e 2. Pela análise do quadro 1 observa-se que todos os artigos selecionados estavam inseridos na base de dados MEDLINE, dentre os quais apenas um estava na língua espanhola, evidenciando uma prevalência de estudos publicados originalmente em inglês $(\mathrm{n}=14)$. 
Quadro 1. Caracterização das publicações quanto ao autor, base de dados, periódico, ano e idioma.

\begin{tabular}{|c|c|c|c|}
\hline AUTORES & $\begin{array}{l}\text { BASE DE } \\
\text { DADOS }\end{array}$ & PERIÓDICO/ANO & IDIOMA \\
\hline Charfi R et al. & MEDLINE & La Tunisie Medicale (2020) & Inglês \\
\hline Gao Y et al. & MEDLINE & Am J Cardiovasc Drugs (2019) & Inglês \\
\hline Gonzalez-Loyola $\mathrm{F}$ et al. & MEDLINE & $\begin{array}{l}\text { European journal of clinical investigation } \\
\qquad(2018)\end{array}$ & Inglês \\
\hline Charnigo R et al. & MEDLINE & $\begin{array}{l}\text { Pacing and Clinical Electrophysiology } \\
\qquad(2018)\end{array}$ & Inglês \\
\hline Lopes R et al. & MEDLINE & $\begin{array}{c}\text { Journal of the American College of } \\
\text { Cardiology (2018) }\end{array}$ & Inglês \\
\hline Yu H et al. & MEDLINE & Circulation Journal (2017) & Inglês \\
\hline García-Iranzo E et al. & MEDLINE & Farmacia Hospitalaria (2017) & Espanhol \\
\hline Adedinsewo D et al. & MEDLINE & Circ Arrhythm Electrophysiol (2017) & Inglês \\
\hline Biteker $\mathrm{M}$ et al. & MEDLINE & J Clin Pharm Ther (2016) & Inglês \\
\hline Van Gelder I et al. & MEDLINE & Lancet (2016) & Inglês \\
\hline $\begin{array}{l}\text { Erath J, Vamos M, } \\
\text { Hohnloser S }\end{array}$ & MEDLINE & Eur Heart J Cardiovasc Pharmacother (2016) & Inglês \\
\hline Al-Zakwani I et al. & MEDLINE & J Cardiovasc Pharmacol Ther (2016) & Inglês \\
\hline Chao $\mathrm{T}$ et al. & MEDLINE & Circulation (2015) & Inglês \\
\hline Okin PM et al. & MEDLINE & J Hypertens (2015) & Inglês \\
\hline Washam J et al. & MEDLINE & The Lancet (2015) & Inglês \\
\hline
\end{tabular}

Conforme o Quadro 2, observa-se que foram encontrados dois estudos observacionais, dois estudos de coorte, quatro análises retrospectivas de dados, dois estudos de incidência, quatro ensaios clínicos controlados e um estudo transversal. 
Quadro 2. Caracterização das publicações quanto ao autor, tipo de estudo e principal achado.

\begin{tabular}{|c|c|c|}
\hline $\begin{array}{c}\text { AUTOR } \\
\text { PRINCIPAL }\end{array}$ & $\begin{array}{l}\text { TIPO DE } \\
\text { ESTUDO }\end{array}$ & PRINCIPAL ACHADO \\
\hline Charfi R et al. & $\begin{array}{c}\text { Estudo } \\
\text { observacional }\end{array}$ & $\begin{array}{l}\text { Os principais efeitos adversos relatados sobre a digoxina foram cardíacos e } \\
\text { digestivos. }\end{array}$ \\
\hline Gao Y et al. & COORTE & $\begin{array}{l}\text { O uso de digoxina foi associado a um aumento por todas as causas de } \\
\text { mortalidade, morte cardiovascular e hospitalização. }\end{array}$ \\
\hline $\begin{array}{l}\text { Gonzalez-Loy } \\
\text { ola F et al. }\end{array}$ & $\begin{array}{l}\text { Análise } \\
\text { retrospectiva de } \\
\text { dados }\end{array}$ & $\begin{array}{l}\text { A digoxina não afetou a mortalidade em IC com FA, mas em pacientes sem } \\
\text { FA os diuréticos e a digoxina foram associados a uma maior mortalidade. }\end{array}$ \\
\hline $\begin{array}{l}\text { Charnigo R et } \\
\text { al. }\end{array}$ & $\begin{array}{c}\text { Análise } \\
\text { retrospectiva de } \\
\text { dados }\end{array}$ & $\begin{array}{l}\text { Os achados sugerem que a digoxina deve ser evitada em pacientes com FA } \\
\text { sem IC anterior, pois aumentou em } 36 \% \text { a mortalidade. }\end{array}$ \\
\hline Lopes R et al. & $\begin{array}{l}\text { Ensaio clínico } \\
\text { controlado }\end{array}$ & $\begin{array}{l}\text { O risco de morte foi relacionado à concentração sérica de digoxina e foi } \\
\text { mais alto em pacientes com concentrações de } 1,2 \mathrm{ng} / \mathrm{ml} \text {. }\end{array}$ \\
\hline Yu H et al. & $\begin{array}{c}\text { Estudo } \\
\text { observacional }\end{array}$ & $\begin{array}{l}\text { O uso de digoxina não foi associado a um risco aumentado de morte em } \\
\text { pacientes com FA. }\end{array}$ \\
\hline $\begin{array}{l}\text { García-Iranzo } \\
\text { E et al. }\end{array}$ & Estudo transversal & $\begin{array}{l}\text { Alguns dos pacientes com FA que faziam o uso crônico da digoxina sem } \\
\text { monitoramento apresentaram episódios de intoxicação digitálica e } \\
\text { subdosagem. }\end{array}$ \\
\hline $\begin{array}{l}\text { Adedinsewo } \\
\text { D et al. }\end{array}$ & $\begin{array}{l}\text { Estudo de } \\
\text { incidência }\end{array}$ & $\begin{array}{l}\text { O uso da digoxina em pacientes com FA foi associado ao maior número de } \\
\text { hospitalizações e mortalidade. }\end{array}$ \\
\hline $\begin{array}{l}\text { Biteker M et } \\
\quad \text { al. }\end{array}$ & $\begin{array}{l}\text { Ensaio clínico } \\
\text { controlado }\end{array}$ & $\begin{array}{c}\text { 60\% dos pacientes com fibrilação atrial faziam o uso impróprio da digoxina. } \\
\text { Sendo de uso mais frequente em pacientes com outras comorbidades } \\
\text { associadas, o que predispõe a maior mortalidade. }\end{array}$ \\
\hline $\begin{array}{l}\text { Van Gelder I } \\
\text { et al. }\end{array}$ & $\begin{array}{l}\text { Ensaio clínico } \\
\text { controlado }\end{array}$ & $\begin{array}{c}\text { Constatou-se que é preciso ter cautela no uso de digoxina em pacientes com } \\
\text { insuficiência renal e que fazem o uso de outras drogas. }\end{array}$ \\
\hline $\begin{array}{l}\text { Erath J, } \\
\text { Vamos M, } \\
\text { Hohnloser S }\end{array}$ & $\begin{array}{l}\text { Estudo de } \\
\text { incidência }\end{array}$ & $\begin{array}{l}\text { Os pacientes com CDI que usaram digitálicos morreram com mais } \\
\text { frequência de causas cardíacas arrítmicas e cardíacas não arrítmicas do que } \\
\text { os pacientes sem digitálicos. }\end{array}$ \\
\hline $\begin{array}{l}\text { Al-Zakwani I } \\
\text { et al. }\end{array}$ & $\begin{array}{l}\text { Análise } \\
\text { retrospectiva de } \\
\text { dados }\end{array}$ & $\begin{array}{l}\text { Digoxina não ofereceu benefícios em relação a tempo de vida para os } \\
\text { pacientes com FA e IC e seu uso em pacientes com IC comparando-se } \\
\text { àqueles que não a utilizaram resultou em maior mortalidade a longo termo. }\end{array}$ \\
\hline Chao $\mathrm{T}$ et al. & COORTE & Digoxina foi associada a maior mortalidade. \\
\hline $\begin{array}{l}\text { Okin PM } \\
\text { okinet al. }\end{array}$ & $\begin{array}{l}\text { Ensaio clínico } \\
\text { controlado }\end{array}$ & $\begin{array}{l}\text { O uso da digoxina em pacientes hipertensos com fibrilação atrial não esteve } \\
\text { associado a maior mortalidade. }\end{array}$ \\
\hline $\begin{array}{l}\text { Washam J et } \\
\text { al. }\end{array}$ & $\begin{array}{c}\text { Análise } \\
\text { retrospectiva de }\end{array}$ & $\begin{array}{c}\text { A digoxina esteve associada a maior mortalidade por todas as causas, morte } \\
\text { súbita e morte vascular. }\end{array}$ \\
\hline
\end{tabular}

Fonte: Dados de pesquisa (2020). 


\section{Discussão}

A presente revisão integrativa foi realizada com o objetivo de analisar a associação da mortalidade com o uso da digoxina no tratamento da fibrilação atrial (FA). Foi demonstrado através de alguns dos estudos selecionados que pacientes que utilizam a digoxina no tratamento de fibrilação atrial possuem um maior risco de morte em relação àqueles que não a utilizam (Adedinsewo et al., 2017; Chao et al., 2015; Gao et al., 2019; Lopes et al., 2018; Washam et al., 2015) e quando comparada a outras classes de medicamentos para o controle da fibrilação atrial, como betabloqueadores e bloqueadores de canal de cálcio, há nela um maior risco de mortalidade (Chao et al., 2015). A alta taxa de letalidade ocorre devido a algumas características da digoxina tais como a sua estreita faixa terapêutica, as interações medicamentosas com outros fármacos e a questão da sua depuração renal, fatores esses que podem provocar o aparecimento de resultados adversos cursando, assim, com o aumento das taxas de mortalidade (Gao et al., 2019).

Observou-se que as pessoas com implante do cardioversor desfibrilador (CDI) que utilizavam digitálicos, como a digoxina, para tratar FA, acabavam morrendo mais por causas cardíacas arrítmicas e não arrítmicas em relação aos que não os utilizavam (Erath, Vamos \& Hohnloser, 2016). Além disso, a digoxina esteve associada a maior mortalidade por todas as causas do estudo (morte súbita e morte vascular), embora não tenha sido encontrado risco em relação a derrame, embolia sistêmica e infarto do miocárdio (Gao et al., 2019). Nesse mesmo sentido, uma análise post-hoc do ensaio ARISTOTLE, demonstrou que se a dosagem for administrada até $1,2 \mathrm{ng} / \mathrm{ml}$ a taxa de mortalidade permanece a mesma, mas ao ultrapassar esse valor, há um aumento considerável de morte entre pacientes, caracterizando um efeito, na mortalidade, intimamente relacionado à dosagem (Lopes et al., 2018).

No que diz respeito aos fatores de risco associados à mortalidade, devido ao uso da digoxina no tratamento da FA, dois dos estudos apontam a disfunção renal como o principal deles. Essa constatação deve-se ao fato de que, quando os pacientes apresentam injúria renal, o fármaco tende a se acumular no corpo fazendo com que sua concentração exceda a dose terapêutica e possa chegar a nível de toxicidade (Gao et al., 2019; Van Gelder, Rienstra, Crijns \& Olshansky, 2016). Além disso, eles relatam a disfunção renal aguda (eGFR $\leq 60 \mathrm{~mL} / \mathrm{min} / 1,73 \mathrm{~m} 2$ ) como um importante determinante para mortalidade por doenças cardíacas e cerebrovasculares em pacientes recebendo terapia com digoxina para FA. Isso porque, a digoxina inibe a bomba de sódio-potássio ATPase e demonstrou também aumentar a mobilização de cálcio plaquetário e a expressão de receptores de ligação a plaquetas. Nesse contexto, o uso de digoxina pode exacerbar a ativação plaquetária pela associação entre a concentração sérica do fármaco e a excreção urinária de 11-desidro-tromboxano B2, um marcador de ativação plaquetária que pode estar associado a um aumento da incidência de doenças cardiovasculares. Esses efeitos são particularmente relevantes em pacientes com FA em tratamento com o fármaco (Lopes et al., 2018).

Outrossim, em um dos estudos foi constatada a relação da maior quantidade de aparecimento de efeitos adversos pelo uso da digoxina em pacientes mais velhos, e observou-se que a concentração plasmática foi significativamente maior em idosos, em comparação aos demais pacientes, apesar de uma menor dose de digoxina ter sido administrada a eles. Assim, isso demonstra uma maior biodisponibilidade da digoxina em adultos mais velhos, uma vez que, o envelhecimento resulta em meiavida de eliminação prolongada devido à menor depuração de digoxina, bem como em diminuição do volume de distribuição do fármaco, uma vez que indivíduos mais velhos, em geral, apresentam baixa massa corporal magra. Nesse contexto, efeitos adversos foram relatados em $47 \%$ desses idosos e em $36 \%$ dos pacientes totais. Entre os efeitos indesejáveis e nocivos, destacaram-se: eventos cardíacos em 29 pacientes (disritmia, bradicardia e distúrbios de condução); problemas digestivos em 28 pacientes (vômitos, náuseas e diarreia) e visuais em 6 mulheres e parestesia em um homem (Charfi et al., 2020).

Ainda nessa perspectiva, um dos achados da revisão foi o fato de pacientes que não tiveram suas concentrações séricas de digoxina regularmente monitoradas apresentarem mais ocorrências hospitalares e mortalidade do que aqueles que eram monitorados. E dentre as causas das hospitalizações dos pacientes não controlados, estavam a intoxicação digitálica e a 
subdosagem (García-Iranzo et al., 2017). Ademais, em um dos estudos foi observado o aumento de hospitalizações por problemas cardiovasculares em indivíduos com FA que faziam a utilização da digoxina (Gao et al., 2019). Dessa maneira, observa-se a importância do acompanhamento e monitorização frequente de pacientes, sob tratamento de FA, que fazem o uso da digoxina, tendo em vista os riscos da superdosagem frente a sua estreita janela terapêutica.

Dos estudos analisados, cinco trouxeram a temática da relação da fibrilação atrial e insuficiência cardíaca associadas ao uso da digoxina. Dessa forma, observou-se que em três desses cinco estudos, os pacientes que apresentavam um quadro de fibrilação atrial, sem história de insuficiência cardíaca (IC), e faziam o uso da digoxina, possuíram maior associação com o aumento da mortalidade por todas as causas. Contudo, nos indivíduos que possuíam histórico de FA e IC, o uso da digoxina não foi relacionado a um aumento da mortalidade (Al-Zakwani et al., 2016; Biteker et al., 2016; Charnigo, Khairy, Guo, Shohoudi, \& Elayi, 2018). Entretanto, uma análise post-hoc do ensaio ENGAGE AF-TIMI 48, relatou exatamente o contrário, ou seja, que entre pacientes com FA e sem IC não houve associação do uso da digoxina com morte cardíaca súbita, porém em indivíduos com FA e IC a utilização do fármaco foi relacionada com todas as causas de morte (Gao et al., 2019). Apenas dois estudos não apresentaram resultados que diferenciam a mortalidade em pacientes com FA com e sem IC que utilizam a digoxina (Yu et al., 2017; Okin P et al., 2015). Desse modo, podemos inferir que dentre os quinze artigos selecionados para análise, apenas dois não encontraram correlação sobre o uso da digoxina no tratamento da FA (com ou sem IC) e aumento da mortalidade. Assim, entende-se que há uma associação intrínseca entre as variáveis analisadas.

\section{Conclusão}

Frente à análise dos estudos supracitados, embora não se tenha um consenso científico acerca do uso da digoxina na terapêutica da fibrilação atrial, pôde-se constatar uma intrínseca relação entre o uso desse fármaco e o incremento da mortalidade em relação a outras classes medicamentosas, como betabloqueadores e inibidores de canais de cálcio. No entanto, não há aquiescência quanto à influência da presença ou não da IC em portadores da FA tratados com digoxina.

Outrossim, houve convergência dos estudos no tangente à prevalência de vários efeitos adversos da digoxina com o avanço da idade; logo, em virtude das alterações fisiológicos ocasionadas pela velhice, há aumento da biodisponibilidade da droga e de seus consequentes danos associados a níveis acima da faixa terapêutica da digoxina. É mister ressaltar também, que pacientes sem monitorização terapêutica foram mais susceptíveis à superdosagem, à intoxicação digitálica e à morte; bem como, a insuficiência renal caracteriza-se como um fator de risco para mortandade, em consequência do acúmulo do fármaco.

Ademais, verificou-se o acréscimo de doenças cardiovasculares acarretadas, possivelmente, pela exacerbação plaquetária induzida pela digoxina. Por fim, verificou-se o maior risco de óbito, por múltiplas causas, tanto em pacientes portadores de CDI quanto os não portadores dessa condição; os quais receberam a digoxina como droga de escolha para FA. Sendo, a mortalidade ainda maior em dosagens superiores a $1,2 \mathrm{ng} / \mathrm{ml}$.

Diante das evidências apresentadas nessa revisão, podemos afirmar que o uso de digoxina em pacientes com FA, portadores ou não de IC, deve ser evitado ou realizado com cautela e acompanhamento rigoroso, posto que essa terapêutica resultou, em muitos casos, no incremento da mortalidade.

A realização de mais estudos, principalmente de ensaios clínicos randomizados, pode vir a elucidar e estabelecer conduta mais firme sobre o dilema do uso da digoxina e aumento de efeitos adversos e mortalidade nos pacientes que usam a droga. Nesse sentido, as pesquisas devem minimizar possíveis vieses, como a prescrição da digoxina para pacientes mais graves ou o surgimento de desfechos desfavoráveis em razão de falha na monitorização terapêutica. Também é interessante a realização de mais estudos que analisem de forma comparativa alternativas farmacológicas para o tratamento da FA, a exemplo dos betabloqueadores. 


\section{Referências}

Adedinsewo, D., Xu, J., Agasthi, P., Oderinde, A., Adekeye, O., Sachdeva, R., \& Onwuanyi, A. (2017). Effect of digoxin use among Medicaid enrollees with atrial fibrillation. Circulation: Arrhythmia and Electrophysiology, 10(5), e004573.

Al-Zakwani, I., Panduranga, P., Zubaid, M., Sulaiman, K., Rashed, W. A., Alsheikh-Ali, A. A., \& Amin, H. (2016). Impact of digoxin on mortality in patients with atrial fibrillation stratified by heart failure: findings from Gulf Survey of Atrial Fibrillation Events in the Middle East. Journal of cardiovascular pharmacology and therapeutics, 21(3), 273-279.

Benjamin, E. J., Muntner, P., Alonso, A., Bittencourt, M. S., Callaway, C. W., Carson, A. P., \& American Heart Association Council on Epidemiology and Prevention Statistics Committee and Stroke Statistics Subcommittee. (2019). Heart disease and stroke statistics-2019 update: a report from the American Heart Association. Circulation, 139(10), e56-e528.

Biteker, M., Başaran, Ö., Dogan, V., Beton, O., Tekinalp, M., Çă̆rı Aykan, A., \& Kırma, C. (2016). Real-life use of digoxin in patients with non-valvular atrial fibrillation: data from the RAMSES study. Journal of clinical pharmacy and therapeutics, 41(6), 711-717.

Chao, T. F., Liu, C. J., Tuan, T. C., Chen, S. J., Wang, K. L., Lin, Y. J., \& Chen, S. A. (2015). Rate-control treatment and mortality in atrial fibrillation. Circulation, 132(17), 1604-1612.

Charfi, R., Sassi, M. B., Gaies, E., Jebabli, N., Daghfous, R., \& Trabelsi, S. (2020). Digoxin Therapeutic Drug Monitoring: Age Influence and Adverse Events. La Tunisie medicale, 98(1), 35-40.

Charnigo, R., Khairy, P., Guo, J., Shohoudi, A., \& Elayi, C. S. (2018). Use of digoxin in atrial fibrillation: One step further in the mortality controversy from the AFFIRM study. Pacing and Clinical Electrophysiology, 41(7), 713-719.

Chugh, S. S., Havmoeller, R., Narayanan, K., Singh, D., Rienstra, M., Benjamin, E. J., \& Murray, C. J. (2014). Worldwide epidemiology of atrial fibrillation: a Global Burden of Disease 2010 Study. Circulation, 129(8), 837-847.

de Moraes, E. R. F. L., Cirenza, C., Lopes, R. D., Carvalho, A. C., Guimaraes, P. O., Rodrigues, A. A. E., \& de Paola, A. A. V. (2019). Prevalence of atrial fibrillation and stroke risk assessment based on telemedicine screening tools in a primary healthcare setting. European journal of internal medicine, $67,36-41$.

Erath, J. W., Vamos, M., \& Hohnloser, S. H. (2016). Effects of digitalis on mortality in a large cohort of implantable cardioverter defibrillator recipients: results of a long-term follow-up study in 1020 patients. European Heart Journal-Cardiovascular Pharmacotherapy, 2(3), 168-174.

Gao, Y., Chang, S., Du, X., Dong, J., Xu, X., Zhou, Y., \& Ma, C. (2019). Association Between Digoxin Use and Adverse Outcomes Among Patients in the Chinese Atrial Fibrillation Registry. American Journal of Cardiovascular Drugs, 19(6), 579-587.

García-Iranzo, E. M., Rodríguez-Lucena, F. J., Matoses-Chirivella, C., García-Monsalve, A., Murcia-López, A. C., \& Navarro-Ruiz, A. (2017). Pharmacokinetic monitoring of chronic treatment with digoxin from Primary Health Care. Farmacia hospitalaria: organo oficial de expresion cientifica de la Sociedad Espanola de Farmacia Hospitalaria, 41(4), 527-532.

Go, A. S., Hylek, E. M., Phillips, K. A., Chang, Y., Henault, L. E., Selby, J. V., \& Singer, D. E. (2001). Prevalence of diagnosed atrial fibrillation in adults: national implications for rhythm management and stroke prevention: the AnTicoagulation and Risk Factors in Atrial Fibrillation (ATRIA) Study. Jama, 285(18), 2370-2375.

Goldberger, Z. D., \& Alexander, G. C. (2014). Digitalis use in contemporary clinical practice: refitting the foxglove. JAMA internal medicine, 174(1), 151154.

Gonzalez-Loyola, F., Abellana, R., Verdú-Rotellar, J. M., Bustamante Rangel, A., Clua-Espuny, J. L., \& Muñoz, M. A. (2018). Mortality in heart failure with atrial fibrillation: Role of digoxin and diuretics. European journal of clinical investigation, 48(11), e13014.

Hindricks, G., Potpara, T., Dagres, N., Arbelo, E., Bax, J. J., Blomström-Lundqvist, C., Boriani, G., Castella, M., Dan, G. A., Dilaveris, P. E., Fauchier, L., Filippatos, G., Kalman, J. M., La Meir, M., Lane, D. A., Lebeau, J. P., Lettino, M., Lip, G., Pinto, F. J., Thomas, G. N., ESC Scientific Document Group (2020). 2020 ESC Guidelines for the diagnosis and management of atrial fibrillation developed in collaboration with the European Association of CardioThoracic Surgery (EACTS). European heart journal, ehaa612. Advance online publication. https://doi.org/10.1093/eurheartj/ehaa612

January, C. T., Wann, L. S., Alpert, J. S., Calkins, H., Cigarroa, J. E., Cleveland, J. C., Jr, Conti, J. B., Ellinor, P. T., Ezekowitz, M. D., Field, M. E., Murray, K. T., Sacco, R. L., Stevenson, W. G., Tchou, P. J., Tracy, C. M., Yancy, C. W., \& ACC/AHA Task Force Members (2014). 2014 AHA/ACC/HRS guideline for the management of patients with atrial fibrillation: executive summary: a report of the American College of Cardiology/American Heart Association Task Force on practice guidelines and the Heart Rhythm Society. Circulation, 130(23), 2071-2104. https://doi.org/10.1161/CIR.0000000000000040

January, C. T., Wann, L. S., Calkins, H., Chen, L. Y., Cigarroa, J. E., Cleveland, J. C., \& Yancy, C. W. (2019). 2019 AHA/ACC/HRS focused update of the 2014 AHA/ACC/HRS guideline for the management of patients with atrial fibrillation: a report of the American College of Cardiology/American Heart Association Task Force on Clinical Practice Guidelines and the Heart Rhythm Society. Journal of the American College of Cardiology, 74(1), $104-132$.

Kornej, J., Börschel, C. S., Benjamin, E. J., \& Schnabel, R. B. (2020). Epidemiology of atrial fibrillation in the 21st century: novel methods and new insights. Circulation Research, 127(1), 4-20.

Lopes, R. D., Rordorf, R., De Ferrari, G. M., Leonardi, S., Thomas, L., Wojdyla, D. M., \& ARISTOTLE Committees and Investigators. (2018). Digoxin and mortality in patients with atrial fibrillation. Journal of the American College of Cardiology, 71(10), 1063-1074.

Moher, D., Liberati, A., Tetzlaff, J., Altman, D. G., \& PRISMA Group (2009). Preferred reporting items for systematic reviews and meta-analyses: the PRISMA statement. PLoS medicine, 6(7), e1000097. https://doi.org/10.1371/journal.pmed.1000097

Rahman, F., Kwan, G. F., \& Benjamin, E. J. (2014). Global epidemiology of atrial fibrillation. Nature Reviews Cardiology, 11(11), 639. 
Research, Society and Development, v. 10, n. 4, e29710414116, 2021

(CC BY 4.0) | ISSN 2525-3409 | DOI: http://dx.doi.org/10.33448/rsd-v10i4.14116

Okin, P. M., Hille, D. A., Wachtell, K., Kjeldsen, S. E., Boman, K., Dahlöf, B., \& Devereux, R. B. (2015). Digoxin use and risk of mortality in hypertensive patients with atrial fibrillation. Journal of hypertension, 33(7), 1480-1486.

Ren, Y., Ribas, H. T., Heath, K., Wu, S., Ren, J., Shriwas, P., \& Kinghorn, A. D. (2020). Na+/K+-ATPase-Targeted Cytotoxicity of (+)-Digoxin and Several Semisynthetic Derivatives. Journal of natural products, 83(3), 638-648.

Somberg J. C. (2014). Digoxin therapy for atrial fibrillation in the twenty-first century. American journal of therapeutics, 21(5), 325-326. https://doi.org/10.1097/MJT.0000000000000153

Van Gelder, I. C., Rienstra, M., Crijns, H. J., \& Olshansky, B. (2016). Rate control in atrial fibrillation. The Lancet, 388(10046), 818-828.

Souza, M. T. D., Silva, M. D. D., \& Carvalho, R. D. (2010). Revisão integrativa: o que é e como fazer. Einstein (São Paulo), 8(1), 102-106.

Washam, J. B., Stevens, S. R., Lokhnygina, Y., Halperin, J. L., Breithardt, G., Singer, D. E., \& Committee, R. A. S. (2015). Digoxin use in patients with atrial fibrillation and adverse cardiovascular outcomes: a retrospective analysis of the Rivaroxaban Once Daily Oral Direct Factor Xa Inhibition Compared with Vitamin K Antagonism for Prevention of Stroke and Embolism Trial in Atrial Fibrillation (ROCKET AF). The Lancet, 385(9985), $2363-2370$.

Yu, H. T., Yang, P. S., Lee, H., You, S. C., Kim, T. H., Uhm, J. S., \& Joung, B. (2017). Outcomes of Rate-Control Treatment in Patients With Atrial Fibrillation and Heart Failure-A Nationwide Cohort Study—. Circulation Journal, CJ-17.

Zoni-Berisso, M., Lercari, F., Carazza, T., \& Domenicucci, S. (2014). Epidemiology of atrial fibrillation: European perspective. Clinical epidemiology, 6, 213. 